TRANSACTIONS OF THE

AMERICAN MATHEMATICAL SOCIETY

Volume 250, June 1979

\title{
DEFORMING TWIST-SPUN KNOTS
}

BY

\author{
R. A. LITHERLAND ${ }^{1}$
}

\begin{abstract}
In [15] Zeeman introduced the process of twist-spinning an $n$-knot to obtain an $(n+1)$-knot, and proved the remarkable theorem that a twist-spun knot is fibred. In [2] Fox described another deformation which can be applied during the spinning process, and which he called rolling. We show that, provided one combines the rolling with a twist, the resulting knot is again fibred. In fact, this result holds for a larger class of deformations, defined below.
\end{abstract}

0. Introduction. The process of spinning may be described as follows. Suppose we have a knotted arc in half 3-space $R_{+}^{3}$, with its endpoints in $R^{2}=\partial R_{+}^{3}$. Rotating $R_{+}^{3}$ about $R^{2}$ generates $R^{4}$, and the arc sweeps out a knotted 2-sphere. Now suppose that during the spinning process we move the arc in $R_{+}^{3}$ (keeping its endpoints fixed); provided it is returned to its original position at the end of the spinning, we again obtain a 2 -sphere in $R^{4}$. We call this process deform-spinning; a precise definition (in any dimension) is given in $\S 1$ (where for convenience we work with the one-point compactification $B^{3}$ of $R_{+}^{3}$ ). The first example of deform-spinning was given by Zeeman [15]; this was the operation of twisting the knot about its axis. Subsequently, Fox [2] described another variation on spinning, called roll-spinning, and showed that this differs from twist-spinning. (Specifically, he showed that the roll-spun figure eight knot cannot be obtained by twist-spinning the figure eight.)

Now, the main result of [15] is the striking theorem that any twist-spun knot is fibred. It is natural to ask whether this can be extended to rolling, or some combination of rolling and twisting. First we need a definition of rolling; in [2] Fox only gives a picture of rolling a figure eight knot, and remarks that the operation resembles "rolling a stocking". Our first observation is that in Fox's pictures points of the arc move from the right of the knot to the left. This suggests that, instead of rolling the knot down the string, we should pull the string through the knot. In other words, we should consider an isotopy of space which moves a base-point once around the knot, and is fixed away from the knot. Unfortunately, this only makes sense for a knotted circle, and we do not get a motion of the corresponding knotted arc,

Received by the editors July 14, 1977.

AMS (MOS) subject classifications (1970). Primary 57C45, 55A25.

Key words and phrases. Twist-spinning, rolling, deformation, fibred knot.

${ }^{1}$ Supported by a grant from the Science Research Council. 
since no point of the knot is fixed throughout. However, we can still use this idea once we observe that in order to define deform-spinning it is enough to know the end result of the motion. This observation is formalised in the discussion preceding Lemma 1.2, and allows us to give an alternative definition of deform-spinning. In $\$ 2$ we give examples using this definition; in particular, we define rolling in Example 2.2. The above discussion should justify this nomenclature; we have not attempted to give a precise definition in terms of a motion of an arc. We remark that in Fox's example the motion induces conjugation by a longitude on the group; this is enough to show that our roll-spun figure eight is the same as his (see §6).

Having arrived at a suitable definition of rolling, we show that an $l$-roll, $m$-twist spun knot is fibred for $m \neq 0$; in fact this is true when rolling is replaced by any of a fairly large class of deformations. The proof occupies $\$ 4$. In $\$ 5$ we investigate the fibre, and in $\$ 6$ we relate the deformations of a classical knot to the automorphisms of its group. In $\$ 7$ we show that (in contrast to twist-spinning simpliciter) the bundle group need not be finite; as a consequence we obtain examples of fibred knots provided by our theorem which cannot be obtained by twist-spinning any knot whatsoever.

Notation. We work in the PL category. All manifolds will be oriented, and all maps between manifolds orientation-preserving. $D^{n}, B^{n}$ will both be used to denote an $n$-ball, and $S^{n}$ an $n$-sphere. The quotient map $\mathbf{R}^{1} \rightarrow S^{1}$ will be written $\theta \rightarrow \bar{\theta}$, where $\bar{\theta}=\bar{\varphi}$ iff $\theta-\varphi \in \mathbf{Z}$. Note that the map $S^{1} \times S^{1} \rightarrow S^{1}$; $\bar{\theta} \times \bar{\varphi} \rightarrow \overline{\theta+\varphi}$ is PL.

An $n$-knot will mean a locally flat pair $\left(S^{n+2}, K\right)$ with $K \cong S^{n}$; we will usually refer to "the knot $K$ ". A ball pair is a locally flat pair $\left(B^{n+2}, \beta\right)$ with $\beta \cong B^{n}$ and $\partial \beta=\beta \cap \partial B^{n+2}$. Again, we will refer to "the knotted ball $\beta$ ".

If $X$ is a space and $g: X \rightarrow X$ a homeomorphism, we write $X \times{ }_{g} S^{1}$ for the space

$$
X \times \mathbf{R} /(x \times \theta=g(x) \times(\theta+1): x \in X, \theta \in \mathbf{R})
$$

which we identify with

$$
X \times I /(x \times 0=g(x) \times 1: x \in X) .
$$

( $I$ is the unit interval $[0,1] \subset \mathbf{R}$.) We denote the equivalence class of $x \times \theta$ by $x \times_{g} \theta$ or $x \tilde{\times} \theta$.

If $G$ is a group and $h: G \rightarrow G$ an automorphism, we denote by $G \times{ }_{h} Z\langle t\rangle$ that extension of $G$ by the infinite cyclic group generated by $t$ in which conjugation by $t$ induces $h$ on $G$. Then

$$
\pi_{1}\left(X \times_{g} S^{1}\right)=\pi_{1}(X) \times_{g_{*}} \mathrm{Z} .
$$


1. Deform-spinning. Let $\left(B^{n+2}, \beta\right)$ be a ball pair. The spin of $\left(B^{n+2}, \beta\right)$ is the sphere pair

$$
\partial\left(B^{n+2}, \beta\right) \times B^{2} \cup_{\partial}\left(B^{n+2}, \beta\right) \times \partial B^{2} .
$$

The notion of deforming the knotted ball $\beta$ during the spinning process may be expressed as follows. Let $f_{\theta}: B^{n+2} \rightarrow B^{n+2}(\theta \in I)$ be an isotopy rel $\partial B^{n+2}$ (i.e. $f_{\theta} \mid \partial B^{n+2}=$ id for all $\theta$ ) such that $f_{1}(\beta)=\beta$; Goldsmith [5] calls $f$ a motion of $\left(B^{n+2}, \beta\right)$. The deform-spun knot corresponding to $f$ is then the sphere pair

$$
\partial\left(B^{n+2}, \beta\right) \times B^{2} \cup_{\partial}\left(B^{n+2} \times \partial B^{2}, \bigcup_{\theta \in I}\left[f_{\theta}(\beta) \times \bar{\theta}\right]\right) .
$$

Whilst this definition corresponds admirably to the intuitive idea of "deforming a knot while it spins", it has the disadvantage that interesting motions may be difficult to describe precisely (cf. Fox [2]). The following reformulation mitigates this problem.

The homeomorphism $g=f^{-1}: B^{n+2} \times I \rightarrow B^{n+2} \times I$ induces a homeomorphism

$$
\bar{g}: B^{n+2} \times \partial B^{2} \rightarrow B^{n+2} \times_{g_{1}} \partial B^{2},
$$

with $\bar{g} \mid \partial B^{n+2} \times \partial B^{2}=$ id. Now $\bar{g}$ sends $\cup_{\theta \in I}\left[f_{\theta}(\beta) \times \bar{\theta}\right]$ to $\cup_{\theta \in I}[\beta \tilde{\times} \bar{\theta}]$ $=\beta \times_{g_{1} \mid} \partial B^{2}$, so extending $\bar{g}$ by the identity we get a homeomorphism from the pair of (1.1) to the pair

$$
\partial\left(B^{n+2}, \beta\right) \times B^{2} \cup_{\partial}\left(B^{n+2}, \beta\right) \times_{g_{1}} \partial B^{2} .
$$

So, for any homeomorphism $g:\left(B^{n+2}, \beta\right) \rightarrow\left(B^{n+2}, \beta\right)$ with $g \mid \partial B^{n+2}=$ id, set

$$
P(g)=\partial\left(B^{n+2}, \beta\right) \times B^{2} \cup_{\partial}\left(B^{n+2}, \beta\right) \times_{g} \partial B^{2} .
$$

Then we have

(1.2) LEMMA. $P(g)$ is a locally flat sphere pair, depending (up to homeomorphism) only on the pseudoisotopy class of $g\left(\mathrm{rel} \partial B^{n+2}\right)$.

Proof. By the Alexander trick, there is an isotopy $F: B^{n+2} \times I \rightarrow B^{n+2} \times$ $I$ with $F \mid \partial B^{n+2} \times I=$ id and $F_{1}^{-1}=g$. As above, this gives a homeomorphism

$$
\partial B^{n+2} \times B^{2} \cup_{\partial} B^{n+2} \times \partial B^{2} \rightarrow \partial B^{n+2} \times B^{2} \cup_{\partial} B^{n+2} \times_{g} \partial B^{2}
$$

But

$$
\partial B^{n+2} \times B^{2} \cup B^{n+2} \times \partial B^{2}=\partial\left(B^{n+2} \times B^{2}\right),
$$

an $(n+3)$-sphere. Similarly $\partial \beta \times B^{2} \cup \beta \times_{g \mid} \partial B^{2}$ is an $(n+1)$-sphere. Local flatness of $P(g)$ follows from that of $\left(B^{n+2}, \beta\right)$. 
Finally, suppose $g$ and $h$ are pseudo-isotopic rel $\partial B^{n+2}$ (as maps of pairs). That is, there is a homeomorphism

$$
H:\left(B^{n+2}, \beta\right) \times I \rightarrow\left(B^{n+2}, \beta\right) \times I
$$

with $H \mid\left(B^{n+2}, \beta\right) \times\{0\}=$ id, $H \mid \partial\left(B^{n+2}, \beta\right) \times I=$ id and $H \mid\left(B^{n+2}, \beta\right) \times$ $\{1\}=h g^{-1}$. Then $H$ induces a homeomorphism

$$
\left(B^{n+2}, \beta\right) \times_{g} \partial B^{2} \rightarrow\left(B^{n+2}, \beta\right) \times_{h} \partial B^{2}
$$

which extends by the identity to $P(g) \cong P(h)$.

Write $H\left(B^{n+2}, \beta\right)$ for the group of self-homeomorphisms $g$ of $\left(B^{n+2}, \beta\right)$ with $g \mid \partial B^{n+2}=$ id, and $\mathscr{D}\left(B^{n+2}, \beta\right)$ for $H\left(B^{n+2}, \beta\right)$ modulo pseudo-isotopy rel $\partial B^{n+2}$. We call elements of $\mathscr{Q}\left(B^{n+2}, \beta\right)$ deformations of $\left(B^{n+2}, \beta\right)$. For $\gamma \in \mathscr{D}\left(B^{n+2}, \beta\right)$ we write $\gamma(\beta)=\left(S^{n+3}, \gamma(\beta)\right)$ for $P(g)$, where $g$ is any representative of $\gamma$, and call the $\operatorname{knot} \gamma(\beta)$ the $\gamma$-spin of $\beta$.

Remarks. (1) The reason for choosing pseudo-isotopy is that it is the strongest congruence relation on $H\left(B^{n+2}, \beta\right)$ through which deform-spinning obviously factors. Using isotopy instead would not affect the considerations of this paper. In case $n=1$, the two relations are the same anyway (see §6).

(2) If $\gamma$ and $\gamma^{\prime}$ are conjugate in $\mathscr{D}\left(B^{n+2}, \beta\right)$ then $\gamma(\beta) \cong \gamma^{\prime}(\beta)$.

Now let $\left(S^{n+2}, K\right)$ be a knot, and fix a point $*$ on $K$. Define $H_{s}\left(S^{n+2}, K\right)$ to be the group of those homeomorphisms $g:\left(S^{n+2}, K\right) \rightarrow\left(S^{n+2}, K\right)$ such that $g=$ id on some neighbourhood of * (depending on $g$ ). For $g, h \in$ $H_{s}\left(S^{n+2}, K\right)$ define $g \sim h$ iff $g$ is pseudo-isotopic to $h$ (as maps of pairs) rel some neighbourhood of $*$. This is a congruence; let $\mathscr{D}\left(S^{n+2}, K\right)=$ $H_{s}\left(S^{n+2}, K\right) / \sim$. (Cf. The "stable homeotopy group" of Giffen [3].) Now suppose that $B_{1}^{n+2}$ is a ball neighbourhood of $*$ such that $\left(B_{1}^{n+2}, B_{1}^{n+2} \cap K\right)$ is unknotted and let $\left(B^{n+2}, \beta\right)$ be the complementary ball pair. There is an evident homomorphism

$$
\varphi: \mathscr{D}\left(B^{n+2}, \beta\right) \rightarrow \mathscr{D}\left(S^{n+2}, K\right)
$$

(extend by the identity). It is not difficult to see that $\varphi$ is an isomorphism, and that for $\gamma \in \mathscr{D}\left(S^{n+2}, K\right), \varphi^{-1}(\gamma)(\beta)$ is independent of the choice of $B_{1}^{n+2}$. [Proof. If $\left({ }^{*} B^{n+2},{ }^{*} \beta\right.$ ) is another pair as above, with $B^{n+2} \subset \operatorname{int}\left({ }^{*} B^{n+2}\right)$, let $\psi: \mathscr{D}\left(B^{n+2}, \beta\right) \rightarrow \mathscr{D}\left({ }^{*} B^{n+2},{ }^{*} \beta\right)$ be the obvious homomorphism. The $\varphi$ 's and $\psi$ 's display $\mathscr{D}\left(S^{n+2}, K\right)$ as a direct limit. But there is a homeomorphism $h$ : $\left(B^{n+2}, \beta\right) \rightarrow\left({ }^{*} B^{n+2},{ }^{*} \beta\right)$ such that $\psi([g])=\left[h g h^{-1}\right]$. Hence $\psi$ is an isomorphism and $\psi(\gamma)\left({ }^{*} \beta\right) \cong \gamma(\beta)$.] We write $\gamma K=\left(S^{n+3}, \gamma K\right)$ for $\varphi^{-1}(\gamma)(\beta)$, the $\gamma$-spin of $K$.

REMARK. (3) The choice of base-point is immaterial, for suppose $*_{1}$ and $*_{2}$ are points of $K$ giving groups $\mathscr{D}_{1}\left(S^{n+2}, K\right)$ and $\mathscr{D}_{2}\left(S^{n+2}, K\right)$. Choose a suitable ball neighbourhood $B_{1}^{n+2}$ of $*_{1} \cup{ }_{2}$, with complementary pair $\left(B^{n+2}, \beta\right)$. We get an isomorphism 


$$
\varphi_{2} \varphi_{1}^{-1}: \mathscr{D}_{1}\left(S^{n+2}, K\right) \rightarrow \mathscr{D}\left(B^{n+2}, \beta\right) \rightarrow \mathscr{D}_{2}\left(S^{n+2}, K\right)
$$

such that $\left(\varphi_{2} \varphi_{1}^{-1}\right)(\gamma)(K) \cong \gamma K$.

\section{Some deformations.}

(2.1) ExAMple. Twist-spinning. Let $\left(S^{n+2}, K\right)$ be a knot. Take a tubular neighbourhood $K \times D^{2}$ of $K$ with exterior $X=\operatorname{cl}\left(S^{n+2}-\left(K \times D^{2}\right)\right)$. (That is, we take an embedding $i: K \times D^{2} \rightarrow S^{n+2}$ extending the projection $K \times$ $\{0\} \rightarrow K$. When no confusion can arise, we identify $K \times D^{2}$ with its image under $i$, and refer to this image as the tubular neighbourhood. In case $n=1$, we always assume $K \times x$ null-homologous in $X$ for $x \in \partial D^{2}$.) Let $\partial X \times I$ be a collar of $\partial X=K \times \partial D^{2}$ in $X$ (with $\partial X$ identified with $\partial X \times\{0\}$; this convention will always apply to collars). Define $t:\left(S^{n+2}, K\right) \rightarrow\left(S^{n+2}, K\right)$ by

$$
\begin{gathered}
t(x \times \bar{\theta} \times \varphi)=x \times(\overline{\theta+\varphi}) \times \varphi, \quad x \times \bar{\theta} \times \varphi \in K \times \partial D^{2} \times I, \\
t(y)=y, \quad y \notin \partial X \times I .
\end{gathered}
$$

Let $\tau$ be the class of $t$ in $\mathscr{D}\left(S^{n+2}, K\right)$. The $\tau^{m}$-spin of $K$ is called the $m$-twist-spin of $K$.

REMARKs. (1) By Remark 3 of $\S 1, \tau$ is independent of the choice of base-point. Suppose $t^{\prime}$ is defined similarly to $t$, but using a different tube and collar. By uniqueness of neighbourhoods and collars (see, for instance, Wall [13] (for neighbourhoods) and Zeeman [14, Chapter 5, Theorem 13] (for collars)) there is a homeomorphism $g: S^{n+2} \rightarrow S^{n+2}$ with $g \mid K=$ id, $g t=t^{\prime} g$. By altering $g$ close to the base-point, $g$ represents an element of $\mathscr{D}\left(S^{n+2}, K\right)$, and still $g t=t^{\prime} g$. Hence $\tau$ is well defined up to conjugacy and, by Remark 2 of $\S 1, \tau^{m}(K)$ is well defined. A similar remark will apply to all our other examples.

(2) Retracing the path from motions to deformations followed in $\$ 1$, one may convince oneself that this definition corresponds to the intuitive idea of twist-spinning introduced by Zeeman in [15]. The proof of Theorem 2.4 will clarify the connection with Zeeman's formal definition.

(3) Note that $t$ is the identity on a neighbourhood of $K$. The following argument, due to Professor R. D. Edwards, shows that every element of $\mathcal{D}\left(S^{n+2}, K\right)$ has a representative of this kind. It suffices to prove this for a ball pair $\left(B^{n+2}, \beta\right)$, so let $g:\left(B^{n+2}, \beta\right) \rightarrow\left(B^{n+2}, \beta\right)$ be a homeomorphism with $g \mid \partial B^{n+2}=$ id. Let $N$ be a regular neighbourhood of $\beta$, with $N \cap \partial B^{n+2}$ a regular neighbourhood of $\partial \beta$ in $\partial B^{n+2}$. Let $N^{\prime}$ be another such, with $N \subset \operatorname{int}\left(N^{\prime}\right)$. Then $\left(g\left(N^{\prime}\right), N^{\prime} \cap \partial B^{n+2}\right)$ is a regular neighbourhood of $(\beta, \partial \beta)$ in $\left(B^{n+2}, \partial B^{n+2}\right)$, so by the theory of relative regular neighbourhoods (Cohen [1, Proposition 7.1(a), Proposition 7.10(a) and Theorem 3.1]) $g$ is isotopic rel $\left(\partial B^{n+2} \cup \beta\right)$ to $g_{1}$ with $g_{1}\left(N^{\prime}\right)=N^{\prime}$. Now define $g_{2}:\left(B^{n+2}, \beta\right) \rightarrow$ $\left(B^{n+2}, \beta\right)$ by 


$$
g_{2}= \begin{cases}g_{1} & \text { on } \operatorname{cl}\left(B^{n+2}-N^{\prime}\right) \cup \partial B^{n+2}, \\ \text { id } & \text { on } N,\end{cases}
$$

and on $N^{\prime}-\operatorname{int}(N) \cong S^{1} \times B^{n+1}$ extend the already defined homeomorphism on the boundary arbitrarily. That this is possible follows from the concordance classification of PL homeomorphisms of $S^{1} \times S^{n}$ (Kato [8] for $n>3$; Gluck [4] for $n=2$; and for $n=1$ use the fact that $g_{1}$ must preserve a longitude of $\beta$, and therefore a meridian of $S^{1} \times B^{2}$ ).

By the Alexander trick, the restrictions of $g_{1}$ and $g_{2}$ to $\left(N^{\prime}, \beta\right)$ are isotopic rel $\partial N^{\prime}$; hence $g_{1}$ and $g_{2}$ are isotopic rel $\partial B^{n+2}$, and so represent the same element of $\mathscr{D}\left(B^{n+2}, \beta\right)$. Thus $g_{2}$ is the required representative.

It also follows that such a representative is unique up to pseudo-isotopy rel $N$. For suppose we have $g_{0}, g_{1}:\left(B^{n+2}, \beta\right) \rightarrow\left(B^{n+2}, \beta\right), g_{i} \mid \partial B^{n+2}=$ id, $g_{i} \mid N=$ id and $g_{0}$ pseudo-isotopic to $g_{1}$ rel $\partial B^{n+2}$. We may assume each $g_{i}=$ id on the larger neighbourhood $N^{\prime}$. Let $G$ be the pseudo-isotopy; i.e.

$$
\begin{gathered}
G:\left(B^{n+2}, \beta\right) \times I \rightarrow\left(B^{n+2}, \beta\right) \times I, \\
G \mid \partial B^{n+2} \times I=\text { id, } G\left|B^{n+2} \times\{0\}=g_{0}, \quad G\right| B^{n+2} \times\{1\}=g_{1} .
\end{gathered}
$$

Then the above argument shows that $G$ is isotopic rel $\partial\left(B^{n+2} \times I\right)$ to $G^{\prime}$ with $G^{\prime} \mid N \times I=\mathrm{id} ; G^{\prime}$ is a pseudo-isotopy from $g_{0}$ to $g_{1} \operatorname{rel}\left(\partial B^{n+2} \cup N\right)$.

(2.2) ExAmple. Roll-spinning. Let $\left(S^{3}, K\right)$ be a 1-knot. Take a tubular neighbourhood $K \times D^{2}$ with exterior $X$, and a collar $\partial X \times I$ of $\partial X$ in $X$. Define $r:\left(S^{3}, K\right) \rightarrow\left(S^{3}, K\right)$ by

$$
\begin{gathered}
r(\bar{x} \times \bar{\theta} \times \varphi)=(\overline{x+\varphi}) \times \bar{\theta} \times \varphi, \quad \bar{x} \times \bar{\theta} \times \varphi \in K \times \partial D^{2} \times I, \\
r(y)=y, \quad y \notin \partial X \times I .
\end{gathered}
$$

(Here we use $K \cong S^{1} \cong \mathrm{R} / \mathrm{Z}$.)

Let $\rho$ be the class of $r$ in $\mathscr{D}\left(S^{3}, K\right)$. The $\rho^{\prime}$-spin of $K$ is called the $l$-roll-spin of $K$.

RemarKs. (1) For the connection between this definition and Fox's original idea, see $\$ 0$.

(2) There is a map $p: X \rightarrow \partial D^{2}$ such that on $\partial X=K \times \partial D^{2}, p$ restricts to projection on the second factor, and such that $p r=p$. This fact allows the extension of Zeeman's theorem to roll-twist-spun knots, and so to other deformations with this property. This motivates the following definitions.

(2.3) Definitions. Let $\left(S^{n+2}, K\right)$ be a knot. A projection for $K$ is a pair $\left(p, K \times D^{2}\right)$ where $K \times D^{2}$ is a tubular neighbourhood of $K$ with exterior $X$, and $p$ is a map $X \rightarrow \partial D^{2}$ extending the projection $K \times \partial D^{2} \rightarrow \partial D^{2}$. A homeomorphism $g:\left(S^{n+2}, K\right) \rightarrow\left(S^{n+2}, K\right)$ is compatible with $\left(p, K \times D^{2}\right)$ if

(i) $g \mid K \times D^{2}=$ id, and

(ii) $p \circ(g \mid X)=p$. 
A deformation $\gamma \in \mathscr{D}\left(S^{n+2}, K\right)$ is untwisted if there is a projection $(p, K \times$ $D^{2}$ ) and a compatible representative of $\gamma$.

We can now state our main theorem.

(2.4) TheOREM. Let $\left(S^{n+2}, K\right)$ be a knot, and let $\gamma \in \mathscr{D}\left(S^{n+2}, K\right)$ be untwisted. For each nonzero integer $m$ the ( $m$-twist)- $\gamma$-spin of $K$ is fibred; i.e. $S^{n+3}-\tau^{m} \gamma K$ is a fibre bundle over $S^{1}$.

The proof will be given in $\$ 4$; first we give more examples of deformations.

(2.5) ExAMPLE. Generalised rolling. Rolling can be extended to $n$-knots $\left(S^{n+2}, K\right)$ as follows. Take a tubular neighbourhood $K \times D^{2}$ of $K$ with exterior $X$, and a collar $\partial X \times I$ of $\partial X$ in $X$. Let $H: K \times I \rightarrow K \times I$ be an isotopy with $H_{0}=H_{1}=$ id. Define $r_{H}:\left(S^{n+2}, K\right) \rightarrow\left(S^{n+2}, K\right)$ by

$$
\begin{gathered}
r_{H}(x \times \bar{\theta} \times \varphi)=H_{\varphi}(x) \times \bar{\theta} \times \varphi, \quad x \times \bar{\theta} \times \varphi \in K \times \partial D^{2} \times I, \\
r_{H}(y)=y, \quad y \notin \partial X \times I .
\end{gathered}
$$

The corresponding deformation $\rho_{H}$ is untwisted as there is a projection $\left(p, K \times D^{2}\right)$ such that $p(y \times \varphi)=p(y)$ for $y \in \partial X, \varphi \in I$, and hence $p r_{H}=$ p.

(2.6) Example. $p$-spun deformations. Let us recall the definition of $p$-spinning a knot $\left(S^{n+2}, K\right)$. (See e.g. Gordon [7, §4].) We fix a point $*$ on $K$, and take a ball neighbourhood $B_{1}^{n+2}$ of $*$ such that $\left(B_{1}^{n+2}, B_{1}^{n+2} \cap K\right)$ is an unknotted ball pair. Let $\left(B^{n+2}, \beta\right)$ be the complementary ball pair. The $p$-spin of $K$, a knot of $S^{n+p}$ in $S^{n+p+2}$, is the pair

$$
\left(S^{n+p+2},{ }^{p} K\right)=\partial\left(B^{n+2}, \beta\right) \times B^{p+1} \cup \cup_{\partial}\left(B^{n+2}, \beta\right) \times \partial B^{p+1} .
$$

If $\gamma \in \mathscr{D}\left(S^{n+2}, K\right)$, we can define a deformation ${ }^{p} \gamma$ of ${ }^{p} K$ as follows. Let $g$ be a representative of $\gamma$ with $g \mid B_{1}^{n+2}=$ id. Define a representative ${ }^{P_{g}}$ of ${ }^{P} \gamma$ by

$$
\begin{aligned}
& p_{g}(x \times y)=x \times y, \quad x \in \partial B^{n+2}, y \in B^{p+1}, \\
& p_{g}(x \times y)=g(x) \times y, \quad x \in B^{n+2}, y \in \partial B^{p+1} .
\end{aligned}
$$

If $\gamma$ is untwisted, so is ${ }^{p} \gamma$. Note that the group $\pi\left({ }^{p} K\right)$ of the $p$-spin of $K$ is equal to the group of $\pi(K)$ of $K$, and $\left({ }^{p} g\right)_{*}=g_{*}$. (The group $\pi(K)$ is $\pi_{1}\left(S^{n+2}-K\right)$.) Hence

$$
\begin{aligned}
\pi\left(\left({ }^{p} \gamma\right)\left({ }^{p} K\right)\right) & \cong \pi\left({ }^{p} K\right) /\left(x=\left({ }^{p} g\right)_{*}(x)\right) \\
& \cong \pi(K) /\left(x=g_{*}(x)\right) \cong \pi(\gamma K) .
\end{aligned}
$$

(2.7) Example. Symmetry-spinning. Suppose the knot $\left(S^{3}, K\right)$ has a symmetry $g$ of order $n \neq 0$; that is, $g$ is a homeomorphism $\left(S^{3}, K\right) \rightarrow\left(S^{3}, K\right)$ of period $n$ with fixed-point set an unknotted circle $J$ disjoint from $K$. Since $g$ moves all points of $K$, it does not represent a deformation. We remedy this by dragging $K$ back to its starting position. 
Formally, we have $S^{3} / g=S^{3}$; let $\alpha: S^{3} \rightarrow S^{3}$ be the quotient map. Let $\bar{K}=\alpha(K), \bar{J}=\alpha(J), \bar{K}$ and $\bar{J}$ are circles in $S^{3}$, with $\bar{J}$ unknotted. Also $\operatorname{Lk}(\bar{K}, \bar{J})=\operatorname{Lk}(K, J)=j$, say, is coprime to $n$. Let $\left(\bar{p}, \bar{K} \times D^{2}\right)$ be a projection for $\bar{K}$, with $\bar{K} \times D^{2}$ disjoint from $\bar{J}$.

Now $\alpha^{-1}\left(\bar{K} \times D^{2}\right)$ is the $n$-fold cyclic cover of $\bar{K} \times D^{2}$; it is therefore a tubular neighbourhood $K \times D^{2}$ of $K$ with

$$
\alpha(\bar{x} \times v)=\overline{n x} \times v \quad\left(\bar{x} \in K, v \in D^{2}\right) .
$$

It follows that $g^{j} \mid K \times D^{2}$, being the canonical covering transformation of $K \times D^{2} \rightarrow \bar{K} \times D^{2}$, is given by

$$
\bar{x} \times v \mapsto(\overline{x+1 / n}) \times v .
$$

Hence, picking an integer $k$ such that $j k \equiv 1(\bmod n)$,

$$
g=g^{j k}: \bar{x} \times v \mapsto(\overline{x+k / n}) \times v, \quad \bar{x} \in K, v \in D^{2} .
$$

Now pick a collar $\partial X \times I$ on the boundary of $X=\operatorname{cl}\left(S^{3}-\left(K \times D^{2}\right)\right)$ and define $s^{\prime}:\left(S^{3}, K\right) \rightarrow\left(S^{3}, K\right)$ by

$$
\begin{aligned}
s^{\prime}(\bar{x} \times \bar{\theta} \times \varphi) & =(\overline{x-(k(1-\varphi) / n)}) \times \bar{\theta} \times \varphi, \\
\bar{x} \times \bar{\theta} \times \varphi \in K \times \partial D^{2} \times I, & (\overline{x-k / n}) \times v, \quad \bar{x} \times v \in K \times D^{2}, \\
s^{\prime}(\bar{x} \times v) & =(y)=y, \quad y \in X-(\partial X \times I) .
\end{aligned}
$$

Then $s^{\prime} g \mid K \times D^{2}=$ id and $s^{\prime} g \mid \operatorname{cl}(X-(\partial X \times I))=g$, so $s_{g, k}=s^{\prime} g$ represents an element $\sigma_{g, k}$ of $\mathscr{D}\left(S^{3}, K\right)$. The projection $\left(p=\bar{p} \alpha, K \times D^{2}\right)$ shows that $\sigma_{g, k}$ is untwisted.

REMARK. This example may also be considered as a generalisation of rolling by taking $n=1, g=\mathrm{id}$ and $J$ any unknot disjoint from $K$. Then $\sigma_{g, k}=\rho^{k}$.

3. Bundles over $S^{1}$. In preparation for the proof of Theorem 2.4 we collect here some facts about bundles over $S^{1}$. In dealing with such bundles we neglect the structure group. Thus a bundle over $S^{1}$ with fibre $F$ is for us a map $q: X \rightarrow S^{1}$ which is locally trivial with fibre $F$, and an equivalence of two such is a homeomorphism $h: X_{1} \rightarrow X_{2}$ of total spaces making

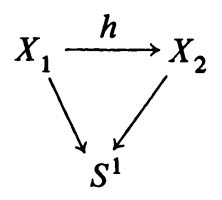

commute. 
To any homeomorphism $g: F \rightarrow F$ there is associated a bundle over $S^{1}$ with fibre $F$; it has total space $F \times_{g} S^{1}$ and projection

$$
q: f \tilde{x} \theta \mapsto \bar{\theta} \quad(f \in F, \theta \in \mathbf{R}) .
$$

Conversely, any bundle $X \rightarrow S^{1}$ with fibre $F$ is equivalent to some $F \times{ }_{g} S^{1}$ $\rightarrow S^{1}$, and then $g$ is called a characteristic map for $X \rightarrow S^{1}$. The bundles associated to homeomorphisms $g_{1}$ and $g_{2}$ are equivalent iff $g_{1}$ is isotopic to a conjugate of $g_{2}$ (lift an equivalence to the infinite cyclic cover $F \times \mathbf{R}$ ). Thus, denoting by $\mathcal{H}(F)$ the homeotopy group of $F$, the order of a characteristic map in $\mathcal{H}(F)$ is an invariant of the equivalence class of a bundle. We call this the order of the bundle. Notice that if the bundle may be endowed with structure group $\mathbf{Z}_{n}$, the order must divide $n$.

If $m \in \mathbf{Z}$, we can define an action of $\mathbf{R}$ on $X=F \times{ }_{g} S^{1}$ by

$$
t \cdot(f \tilde{\times} \theta)=f \tilde{\times}(\theta+m t) \quad(t \in \mathbf{R}, f \tilde{x} \theta \in X) .
$$

This satisfies

$$
q(t \cdot x)=q(x)+\overline{m t} \quad(t \in \mathbf{R}, x \in X) .
$$

We prove a converse to this.

(3.2) LeMMA (CF. ZEEMAN [15, LeMMA 5]). Let $m$ be a nonzero integer. If $q$ : $X \rightarrow S^{1}$ is a map onto $S^{1}$ and if $R$ acts on $X$ so that (3.1) holds, then $q: X \rightarrow S^{1}$ is a fibre bundle with characteristic map given by the action of $-(1 / m) \in \mathbf{R}$.

Proof. Let $F=q^{-1}(\overline{0})$, and let $g: F \rightarrow F$ be the homeomorphism $f \mapsto(-1 / m) \cdot f$. Then we can define $\varphi: F \times{ }_{g} S^{1} \rightarrow X$ by $\varphi(f \tilde{\times} \theta)=$ $(\theta / m) \cdot f$. Now let $q^{\prime}: X \rightarrow \mathbf{R}$ be any function (not continuous) such that $\overline{q^{\prime}(x)}=q(x)$ for each $x \in X$. Define $x: X \rightarrow F \times{ }_{g} S^{1}$ by

$$
\chi(x)=\left[\left(-q^{\prime}(x) / m\right) \cdot x\right] \tilde{\times} q^{\prime}(x) \text {. }
$$

If $q^{\prime \prime}: X \rightarrow \mathbf{R}$ also lifts $q$, then $q^{\prime \prime}(x)=q^{\prime}(x)+n_{x}$ for some integer $n_{x}$. Hence

$$
\begin{aligned}
{\left[\left(-q^{\prime \prime}(x) / m\right) \cdot x\right] \tilde{\times} q^{\prime \prime}(x) } & =\left[\left(-n_{x} / m\right) \cdot\left(-q^{\prime}(x) / m\right) \cdot x\right] \tilde{\times} q^{\prime \prime}(x) \\
& =g^{\left(n_{x}\right)}\left[\left(-q^{\prime}(x) / m\right) \cdot x\right] \tilde{\times} q^{\prime \prime}(x) \\
& =\left[\left(-q^{\prime}(x) / m\right) \cdot x\right] \tilde{\times}\left(q^{\prime \prime}(x)-n_{x}\right) \\
& =\left[\left(-q^{\prime}(x) / m\right) \cdot x\right] \tilde{\times} q^{\prime}(x)
\end{aligned}
$$

and so $\chi$ is independent of the choice of $q^{\prime}$.

But we may cover $S^{1}$ by sets $U$ such that $q^{\prime}$ can be taken PL on $q^{-1}(U)$; hence $\chi$ is PL on $X$. Then $\varphi$ and $\chi$ are mutually inverse, and

$$
q \varphi(f \tilde{\times} \theta)=q(f)+\bar{\theta}=\bar{\theta} \quad(f \in F, \theta \in \mathbf{R}),
$$

so $\varphi$ is a bundle equivalence. 
4. Proof of Theorem 2.4. Recall that we are given a knot $\left(S^{n+2}, K\right)$ and an untwisted deformation $\gamma \in \mathscr{D}\left(S^{n+2}, K\right)$. We have to prove that $\tau^{m} \gamma K$ is fibred for $m \neq 0$.

Let $\left(p, K \times D^{2}\right)$ be a projection for $K$, and $g$ a compatible representative for $\gamma$. Let $X=\operatorname{cl}\left(S^{n+2}-\left(K \times D^{2}\right)\right)$ be the exterior of $K$, and take a collar $\partial X \times I$ of $\partial X$ in $X$. Let $t$ be the representative of $\tau$ defined using $\partial X \times I$ as in Example 2.1.

Take an $n$-ball neighbourhood $K_{-}$of the base-point in $K$, and set $B_{-}=$ $K_{-} \times D^{2}$. Then $\left(B_{-}, K_{-}\right)$is a standard ball pair, so we can construct $\tau^{\bar{m}} \gamma K$ using the complementary ball pair $\left(B_{+}, K_{+}\right)$. Thus

$$
\left(S^{n+3}, \tau^{m} \gamma K\right)=\partial\left(B_{+}, K_{+}\right) \times B^{2} \cup_{\partial}\left(B_{+}, K_{+}\right) \times_{\left(t m_{g}\right)} \partial B^{2} .
$$

Note that

$$
\partial B_{+}=\partial B_{-}=\partial K_{+} \times D^{2} \cup K_{-} \times \partial D^{2}
$$

(see Figure 1). A tubular neighbourhood of $\tau^{m} \gamma K$ is $\partial K_{+} \times D^{2} \times B^{2} \cup K_{+}$ $\times D^{2} \times \partial B^{2}$ and so $\tau^{m} \gamma K$ has exterior

$$
X^{\prime}=K_{-} \times \partial D^{2} \times B^{2} \cup X \times_{\left(l^{m}\right)} \partial B^{2} .
$$

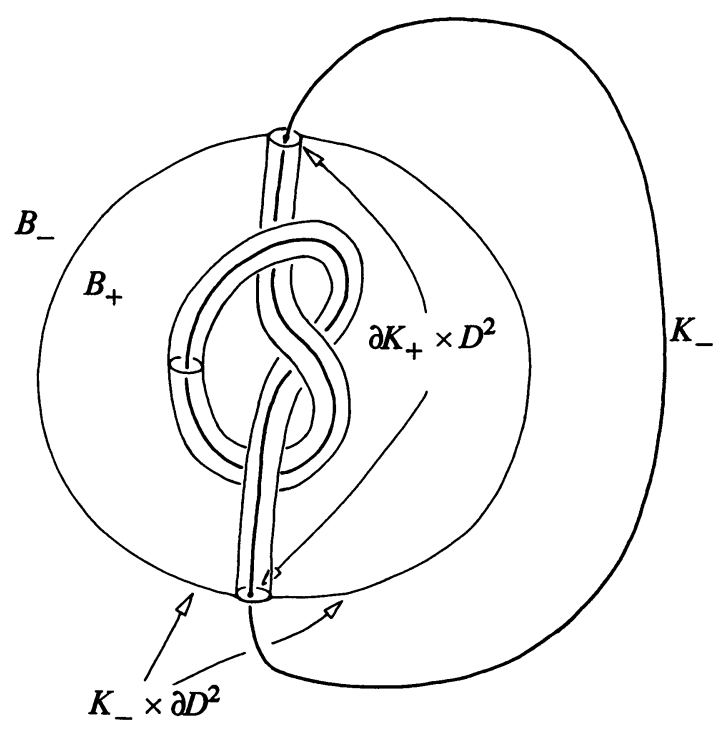

FIgURE 1

We shall fibre $X^{\prime}$.

Now let $F: \partial X \times I \rightarrow \partial X \times I$ be the isotopy

$$
x \times \bar{\theta} \times \varphi \mapsto x \times(\overline{\theta+m \varphi}) \times \varphi, \quad x \times \bar{\theta} \in K \times \partial D^{2}, \varphi \in I .
$$


Then $F$ extends to an isotopy $H: X \times I \rightarrow X \times I$ with $H_{1}=t^{-m}$. [Proof. Let $\mu: I \times I \rightarrow I$ be a map with $\mu(\varphi, 0)=\mu(0, \varphi)=0, \mu(\varphi, 1)=\mu(1, \varphi)=\varphi$; e.g. $\mu(\varphi, \psi)=\max (0, \varphi+\psi-1)$. Define $H$ by

$$
\begin{gathered}
(y \times \varphi) \times \psi \mapsto F_{\mu(1-\varphi, \psi)}(y) \times \varphi \times \psi, \quad y \times \varphi \in \partial X \times I, \psi \in I, \\
z \times \psi \mapsto z \times \psi, \quad z \in X-(\partial X \times I), \psi \in I .
\end{gathered}
$$

This has the required properties.]

$H$ induces a homeomorphism

$$
\bar{H}: X \times_{\left(t^{m}\right)} \partial B^{2} \rightarrow X \times_{g} \partial B^{2} .
$$

Extending by the identity gives a homeomorphism

$$
X^{\prime} \cong K_{-} \times \partial D^{2} \times B^{2} \cup_{\alpha} X \times_{g} \partial B^{2}
$$

where $\alpha$ is the restriction of $\bar{H}$ to $K_{-} \times \partial D^{2} \times \partial B^{2}$, and is thus given by the formula

$$
x \times \bar{\theta} \times \bar{\varphi} \mapsto x \times(\overline{\theta+m \varphi}) \times \bar{\varphi} .
$$

In case $g=\mathrm{id}$, note the resemblance of (4.1) and (4.2) to Zeeman's definition of twist-spinning; the argument above can be used to show that the two definitions agree. From here on we follow closely Zeeman's proof.

Let us set $Y=X \times_{g} \partial B^{2}$. First we define a map $q: Y \rightarrow \partial D^{2}$ by

$$
q(y \tilde{\times} \varphi)=p(y)-\overline{m \varphi}, \quad y \in X, \varphi \in \mathbf{R} .
$$

This is well defined as $p g=p$ on $X$. Secondly, we define an action of $\mathbf{R}$ on $Y$ :

$$
t \cdot(y \tilde{\times} \varphi)=y \tilde{\times}(\varphi-t), \quad t \in \mathbf{R}, y \in X, \varphi \in \mathbf{R} \text {. }
$$

Then

$$
q(t \cdot(y \tilde{\times} \varphi))=p(y)-(\overline{m(\varphi-t)})=q(y \tilde{\times} \varphi)+\overline{m t} .
$$

Thus Lemma 3.2 applies to show that $q: Y \rightarrow \partial D^{2}$ is a fibre bundle. Now the composite map

$$
K_{-} \times \partial D^{2} \times \partial B^{2} \stackrel{\alpha}{\rightarrow} \partial X \times_{g} \partial B^{2} \stackrel{q}{\rightarrow} \partial D^{2}
$$

sends $x \times \bar{\theta} \times \bar{\varphi}$ to

$$
p(x \times(\overline{\theta+m \varphi}))-\overline{m \varphi}=\overline{\theta+m \varphi}-\overline{m \varphi}=\bar{\theta} ;
$$

i.e. it is projection on the $\partial D^{2}$ factor. Hence $q$ extends to a fibre bundle $q$ : $X^{\prime} \rightarrow \partial D^{2}$. Moreover, it is straightforward to check that on $\partial X^{\prime} \simeq \tau^{m} \gamma K$ $\times \partial D^{2}, q$ is projection on the $\partial D^{2}$ factor. Hence the fibration extends nicely over $\tau^{m} \gamma K \times\left(D^{2}-0\right) \cong \tau^{m} \gamma K \times \partial D^{2} \times[0,1)$, and the theorem is proved. 
REMARK. From the proof, the fibre is homeomorphic to the interior of

$$
q^{-1}(\overline{0})=K_{-} \times\{\overline{0}\} \times B^{2} \cup_{\alpha \mid}\left\{y \tilde{\times} \varphi \in X \times_{g} \partial B^{2} \mid p(y)=\overline{m \varphi}\right\} .
$$

Thus the fibre is $\operatorname{punc}(M)=M-\{\mathrm{pt}\}$, where

$$
M=K \times B^{2} \cup_{\beta}\left\{y \tilde{\times} \varphi \in X \times{ }_{g} S^{1} \mid p(y)=\overline{m \varphi}\right\}
$$

for

$$
\beta:\left\{\begin{array}{l}
K \times \partial B^{2} \stackrel{\simeq}{\rightarrow}\left\{y \tilde{\times} \varphi \in \partial X \times{ }_{g} S^{1} \mid p(y)=\overline{m \varphi}\right\}, \\
x \times \bar{\varphi} \mapsto(x \times \overline{m \varphi}) \tilde{\times} \varphi .
\end{array}\right.
$$

Also the characteristic map is given on $q^{-1}(\overline{0}) \cap Y$ by the action of $-1 / m$, namely $y \tilde{\times} \varphi \mapsto y \tilde{\times}(\varphi+1 / m)$. Under $\alpha$ this corresponds to

$$
\left\{\begin{array}{l}
K_{-} \times\{\overline{0}\} \times \partial B^{2} \rightarrow K_{-} \times\{\overline{0}\} \times \partial B^{2}, \\
x \times \overline{0} \times \bar{\varphi} \mapsto x \times \overline{0} \times(\overline{\varphi+1 / m}) .
\end{array}\right.
$$

Denote by $\operatorname{rot}(\bar{\theta}): B^{2} \rightarrow B^{2}$ the conewise extension of the map $\left(\partial B^{2} \rightarrow \partial B^{2}\right.$; $\bar{\varphi} \mapsto \overline{\varphi+\theta}$ ) for any $\bar{\theta} \in \partial B^{2}$. Then the extension

$$
\left\{\begin{array}{l}
K_{-} \times\{\overline{0}\} \times B^{2} \rightarrow K_{-} \times\{\overline{0}\} \times B^{2}, \\
x \times \overline{0} \times v \mapsto x \times \overline{0} \times \operatorname{rot}(\overline{1 / m})(v)
\end{array}\right.
$$

of (4.3) is a characteristic map for the bundle $K_{-} \times \partial D^{2} \times B^{2} \rightarrow \partial D^{2}$. It follows that a characteristic map for the bundle provided by the theorem is the restriction of

$$
\left\{\begin{array}{l}
M \rightarrow M, \\
{[x \times v \mapsto x \times \operatorname{rot}(\overline{1 / m})(v)] \cup[y \tilde{\times} \varphi \mapsto y \tilde{\times}(\varphi+1 / m)] .}
\end{array}\right.
$$

5. The fibre. In this section we describe the fibre of $\tau^{m} \gamma K$ in more recognisable terms than those of the remark of $\$ 4$ when $\gamma$ is one of the deformations defined in Examples 2.2, 2.5 and 2.7. One may easily show, using Remark 3 of 2.1 , that $\tau^{m} \gamma K \cong-\tau^{-m} \gamma^{-1} K$, so we need only consider $m>0$.

(5.1) Proposition. Let $\left(S^{n+2}, K\right)$ be an n-knot, and $H: K \times I \rightarrow K \times I$ an isotopy with $H_{0}=H_{1}=$ id. Let $G: K \times \partial D^{2} \rightarrow K \times \partial D^{2}$ be the homeomorphism $x \times \bar{\theta} \rightarrow H_{\theta}(x) \times \bar{\theta}$. Let $N^{n+2}$ be the manifold obtained from the $m$-fold cyclic branched cover of $K$ by removing a tubular neighbourhood of the lift of $K$ and sewing it back using $G$. Then the fibre of $\tau^{m} \rho_{H} K$ is a punctured $N$, and its closure is a bounded punctured $N$ with boundary the knot $\tau^{m} \rho_{H} K$. Moreover, off a neighbourhood of the lift of $K$ the characteristic homeomorphism is equal to the canonical covering homeomorphism. $\left(\rho_{H}\right.$ is defined in Example 2.5.) 
Proof. We will use the notations of (2.5) and of $\$ 4$. By the remark of $\$ 4$ it suffices to identify $N$ with

$$
M=K \times B^{2} \cup_{\beta}\left\{y \tilde{\times} \varphi \in X \times{ }_{\left(r_{H}\right)} S^{1} \mid p(y)=\overline{m \varphi}\right\} .
$$

Now the $m$-fold cover of $X$ may be obtained by pulling back the $m$-fold cover $\left(S^{1} \rightarrow S^{1} ; \bar{\varphi} \rightarrow \overline{m \varphi}\right)$ along $p$; thus it is

$$
\tilde{X}=\left\{y \times \bar{\varphi} \in X \times S^{1} \mid p(y)=\overline{m \varphi}\right\} .
$$

The $m$-fold branched cover of $K$ is then $K \times B^{2} \cup_{\beta^{\prime}} \tilde{X}$, where $\beta^{\prime}$ is the homeomorphism

$$
\left\{\begin{array}{l}
K \times \partial B^{2} \rightarrow\left\{y \times \bar{\varphi} \in \partial X \times S^{1} \mid p(y)=\overline{m \varphi}\right\} \\
x \times \bar{\varphi} \mapsto(x \times \overline{m \varphi}) \times \bar{\varphi}
\end{array}\right.
$$

We can define a homeomorphism

$$
\Phi:\left\{y \tilde{x} \varphi \in X \times_{\left(r_{H}\right)} S^{1} \mid p(y)=\overline{m \varphi}\right\} \rightarrow \tilde{X}
$$

with $\Phi \beta=\beta^{\prime} G$ as follows. Define an isotopy $\Psi: X \times I \rightarrow X \times I$ by

$$
\begin{gathered}
(x \times \bar{\theta} \times \varphi) \times \psi \mapsto\left(H_{\psi} H_{\mu(\varphi, \psi)}^{-1}(x) \times \bar{\theta} \times \varphi\right) \times \psi, \\
\quad x \times \bar{\theta} \times \varphi \in \partial X \times I, \psi \in I, \\
y \times \psi \mapsto y \times \psi, \quad y \in X-(\partial X \times I), \psi \in I .
\end{gathered}
$$

(Recall $\mu: I \times I \rightarrow I$ with $\mu(\varphi, 0)=\mu(0, \varphi)=0, \mu(\varphi, 1)=\mu(1, \varphi)=\varphi$.) Then $\Psi_{1}=r_{H}^{-1}$, so $\Psi$ induces a homeomorphism $X \times{ }_{\left(r_{H}\right)} S^{1} \rightarrow X \times S^{1}$. Moreover, $p \Psi_{\psi}=p$ for all $\psi \in I$, so this restricts to a homeomorphism

$$
\Phi:\left\{y \tilde{\times} \varphi \in X \times_{\left(r_{H}\right)} S^{1} \mid p(y)=\overline{m \varphi}\right\} \rightarrow \tilde{X} .
$$

For $x \times \bar{\theta} \in K \times \partial B^{2}$,

$$
\begin{aligned}
\Phi \beta(x \times \bar{\theta}) & =\Phi((x \times \overline{m \theta} \times 0) \tilde{\times} \theta) \\
& =\left(H_{\theta}(x) \times \overline{m \theta} \times 0\right) \times \bar{\theta}=\beta^{\prime} G(x \times \bar{\theta}) .
\end{aligned}
$$

Hence the fibre is as claimed. Also the characteristic homeomorphism is equal to the covering transformation on the lift of $X-(\partial X \times I)$.

(5.2) COROLlary. Let $\left(S^{3}, K\right)$ be a 1-knot. The fibre of $\tau^{m} \rho^{l} K$ is the $m$-fold cyclic cover of $S^{3}(K, m / l)$ branched over $K$. ( $\rho$ is defined in Example 2.2.)

Proof. By $S^{3}(K, m / l)$ we mean the manifold obtained from $S^{3}$ by removing a tubular neighbourhood $K \times D^{2}$ of $K$ and sewing it back by means of a homeomorphism $K \times \partial D^{2} \rightarrow K \times \partial D^{2}$ which takes a meridian to $(a \cdot$ meridian $)+(b \cdot$ longitude $)$; here $a$ and $b$ are coprime integers with 
$a / b=m / l$. This notation for Dehn surgery is taken from Rolfsen [10, Chapter 9].

In [6], Goldsmith shows how to commute the operations of surgery and taking a branched cover. Using this, the result is immediate from (5.1). (Note: Goldsmith considers only surgery on a link disjoint from the branch set. Her proof goes through unchanged without this hypothesis.)

Note that we use the term "branched cyclic cover" rather loosely, in that the projection is not required to be 1-1 over the branch set; it will be 1-1 iff $m$ divides $l$. Also if $m$ and $l$ are coprime the branching index is 1 ; i.e. we have an unbranched cover.

In case $m=1$ in (5.2), the fibre of $\tau \rho^{l} K$ is just $S^{3}(K, 1 / l)$, punctured on the knot $K$, and the characteristic homeomorphism is equal to the identity off a neighbourhood of $K$. In fact, using the proof of (5.1) one can show that the characteristic homeomorphism is isotopic to the identity on $S^{3}(K, 1 / l)$ by an isotopy which rotates $K$ on itself $l$ times. From this it is not difficult to prove the following result, which will be used in $\$ 7$.

(5.3) Corollary. If $\left(S^{3}, K\right)$ is a $1-k n o t, \tau \rho^{l} K$ has group $\pi_{1} S^{3}(K, 1 / l) \times \mathbf{Z}$. $A$ meridian of $\tau \rho^{l} K$ is the product of a generator of $\mathbf{Z}$ and the image of a meridian of $K$ under the map $\pi_{1}\left(S^{3}-K\right) \rightarrow \pi_{1} S^{3}(K, 1 / l)$.

(5.4) Proposition. Let $\left(S^{3}, K\right)$ be a knot with a symmetry $g$ of order $n$. Let $\bar{K}, \bar{J}, k$ and $\sigma_{g, k}$ be as in Example 2.7. Let $N^{3}$ be the mn-fold cyclic cover of $S^{3}(\bar{K}, m / k)$ branched over $\bar{K} \cup \bar{J}$ which corresponds to the kernel of the homomorphism

$$
\pi_{1}\left(S^{3}-\bar{K} \cup \bar{J}\right) \stackrel{\text { abelianise }}{\rightarrow} \mathbf{Z}\left\langle t_{1}\right\rangle \times \mathbf{Z}\left\langle t_{2}\right\rangle \rightarrow \mathbf{Z}_{m n}\langle t\rangle .
$$

Here $t_{1}$ corresponds to a meridian of $\bar{K}, t_{2}$ to a meridian of $\bar{J}$, and the last homomorphism sends $t_{1}$ to $t, t_{2}$ to $t^{-m}$.

Then the fibre of $\tau^{m} \sigma_{g, k} K$ is punc $(N)$, and off a neighbourhood of the lift of $\bar{K}$ the characteristic homeomorphism and the canonical covering transformation agree.

Proof. We use the notations of (2.7) and of $\$ 4$. It suffices to identify $N$ with

$$
M=K \times B^{2} \cup_{\beta}\left\{y \tilde{\times} \varphi \in X \times_{\left(s_{, \alpha}\right)} S^{1} \mid p(y)=\overline{m \varphi}\right\} .
$$

As in (5.1), the second term is homeomorphic to

$$
\left\{y \tilde{\times} \varphi \in X \times{ }_{g} S^{1} \mid p(y)=\overline{m \varphi}\right\}
$$

(as $s_{g, k}$ is isotopic to $g$ on $X$ ). We shall show that

$$
\left\{y \tilde{\times} \varphi \in(X-J) \times_{g} S^{1} \mid p(y)=\overline{m \varphi}\right\}
$$


is the (unbranched) cover of $\alpha(X-J)=S^{3}-\left(\operatorname{int}\left(\bar{K} \times D^{2}\right) \cup \bar{J}\right)$ defined in the statement of the proposition.

Let $\bar{X}=\alpha(X)$. Consider the following diagram, in which $q$ is projection on the first factor, $r$ is the quotient map and $\alpha^{\prime}$ is defined by commutativity.

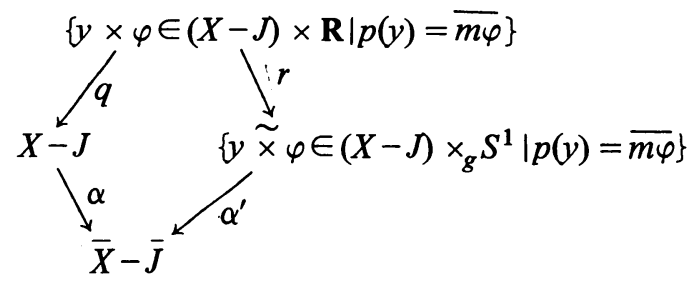

The map $\alpha q$ is a regular cover whose deck group $G$ is $\mathbf{Z}\langle$ id $\times \operatorname{tr}\rangle \oplus \mathbf{Z}_{n}\langle g \times$ id $\rangle$, where tr: $\mathbf{R} \rightarrow \mathbf{R}$ is translation by $1 / m$; the map $\pi_{1}(\bar{X}-\bar{J}) \rightarrow G$ is given by $t_{1} \mapsto$ id $\times \operatorname{tr}, t_{2} \mapsto g \times$ id. Now $\left\{y \tilde{\times} \varphi \in(X-J) \times{ }_{g} S^{1} \mid p(y)=\overline{m \varphi}\right\}$ is obtained by quotienting by the subgroup $\left\langle g \times \operatorname{tr}^{m}\right\rangle$ of $G$. Thus $\alpha^{\prime}$ is a regular cover; its deck group is $G /\left\langle g \times \operatorname{tr}^{m}\right\rangle \cong \mathbf{Z}_{m n}\langle t\rangle$, the map $G \rightarrow \mathbf{Z}_{m n}\langle t\rangle$ being id $\times \operatorname{tr} \mapsto t, g \times \mathrm{id} \mapsto t^{-m}$. This is the cover it was claimed to be.

The remainder of the proof is similar to (5.1) and (5.2), and is omitted.

6. Deformations of classical knots. In this section we show, using results of Waldhausen, that the group of deformations of a nontrivial knot $K$ in $S^{3}$ is determined by the group system of $K$. Let $G$ be the group of $K$ and choose a peripheral subgroup $G_{\partial}$. Define

$$
\operatorname{Aut}_{\partial}(G)=\left\{\alpha \in \operatorname{Aut}(G)|\alpha| G_{\partial}=\mathrm{id}\right\} .
$$

Since any two peripheral subgroups are conjugate, this is independent (up to isomorphism) of the choice of $G_{\partial}$.

(6.1) THEOREM. $\mathscr{D}\left(S^{3}, K\right) \cong \mathrm{Aut}_{2} G$.

Proof. Let $X$ be the exterior of a tubular neighbourhood of $K$. Take a base-point $x_{0}$ on $\partial X$, and let

$$
\left(G, G_{\partial}\right)=\left(\pi_{1}\left(X, x_{0}\right), \pi_{1}\left(\partial X, x_{0}\right)\right) .
$$

Let $\mathcal{H}(X, \partial X)$ be the group of homeomorphisms $g: X \rightarrow X$ with $g \mid \partial X=$ id, modulo pseudo-isotopy rel $\partial X$.

(6.2) Lemma. The homomorphism $\mathcal{H C}(X, \partial X) \rightarrow \mathrm{Aut}_{\partial} G ;[g] \rightarrow g_{*}$ is an isomorphism.

Proof of Lemma. This follows from the work of Waldhausen [12] in a similar manner to Corollary 7.5 of [12]. Note that $[g]$ determines $g_{*}$ uniquely, not just up to inner automorphism, as the base-point is on $\partial X$. We remark that Lemma 6.3 of [12] only works if base-points are taken in the interior. The 
crucial point is when, having constructed a map $f: M \rightarrow N$ inducing a given homomorphism on $\pi_{1}$, a boundary component of $M$ is homotoped into $\partial N$. If the base-point is on this boundary component, to do this rel base-point requires more than that $f_{*}$ preserve the peripheral structure. In our case this still works because $G$ is sent to itself, not just to a conjugate. The details are left to the reader.

Note that pseudo-isotopy could be replaced by isotopy (or by homotopy).

Now Remark 3 of (2.1) shows that the homomorphism $\mathcal{H}(X, \partial X) \rightarrow$ $\mathscr{D}\left(S^{3}, K\right)$ given by extending by the identity is an isomorphism, and the theorem is proved.

Notice that if $\mu$ is a meridian and $\lambda$ a longitude generating $G_{\partial}=\mathbf{Z} \oplus \mathbf{Z}, \tau$ corresponds to conjugation by $\mu$ and $\rho$ to conjugation by $\lambda$. (It follows from Simon [11] that these are the only inner automorphisms in $\mathrm{Aut}_{\partial} G$.) If $K$ is not a torus knot, $G$ has trivial centre, so we have proved

(6.3) CoRollary. If $K$ is not a torus knot, the subgroup of $\mathscr{D}\left(S^{3}, K\right)$ generated by $\tau$ and $\rho$ is isomorphic to $\mathbf{Z} \oplus \mathbf{Z}$.

Let $\kappa_{p, q}$ be the torus knot of type $(p, q)$. For $K=\kappa_{p, q} G$ has infinite cyclic centre generated by $\mu^{p q} \lambda$. Thus we have

(6.4) Corollary. In $\mathscr{D}\left(S^{3}, \kappa_{p, q}\right), \tau^{p q} \rho=1$.

The fact that $\mu^{p q} \lambda$ is in the centre of $\pi\left(\kappa_{p, q}\right)$ is a consequence of the existence of a fibring of $\kappa_{p, q}$ with bundle group $\mathbf{Z}_{p q}$. (An excellent description of this fibring for the trefoil $\kappa_{2,3}$ may be found in [15].) Corollary 6.4 may be derived directly from this as follows. The fibring gives rise to an $S^{1}$-action on $S^{3}-\kappa_{p, q}$. Define an isotopy of $\left(S^{3}, \kappa_{p, q}\right)$ by using the $S^{1}$-action outside a tubular neighbourhood of $\kappa_{p, q}$, holding fixed a smaller tube, and filling in suitably on the intervening collar. The endpoint of this isotopy represents $\tau^{p q} \rho$, which is thus trivial.

In fact, using the presentation $G \cong\left|a, b: a^{p}=b^{q}\right|$ of the group of $\kappa_{p, q}$ and taking $G_{\partial}=\left\langle a^{p}, b^{r} a^{s}\right\rangle(p r+q s=1)$, it is not hard to show that $\mathrm{Aut}_{\partial} G$ is generated by conjugation by $\mu=b^{r} a^{s}$, and so to deduce

(6.5) CoRollary. $\mathscr{D}\left(S^{3}, \kappa_{p, q}\right) \cong \mathbf{Z}$, generated by $\tau$.

7. Knots with infinite bundle group. In the last section we saw that deformations of the type considered in Theorem 2.4 and distinct from $m$-twisting do exist. It is conceivable that these might give rise only to knots obtainable by twist-spinning; in this section we show that this is not the case. The method is to determine which powers of a meridian lie in the centre of a knot group, and was suggested by [7]. 
If $G$ is a group, $C(G)$ will denote its centre. Let $\left(S^{n+2}, K\right)$ be a knot, $G$ its group and $a \in G$ a meridian. Then $C(G) \cap\langle a\rangle=\left\langle a^{k}\right\rangle$ for a unique nonnegative integer $k$, namely the order of $a$ in $G / C(G)$. Since any two meridians are conjugate in $G, k$ is independent of the choice of $a$; we write $c(K)$ for $k$. Clearly, $c(K)$ is an invariant of knot type. (In fact, more is true; in [7] Gordon shows that $c(K)$ is an invariant of the homotopy type of a fibred knot complement, subject to certain restrictions on the homotopy of the fibre.)

(7.1) LEMMA. If $K$ is a fibred knot with a fibring of order $m$, then $c(K)$ divides $m$.

Proof. Let $X$ be an exterior of $K$. Then $X$ is fibred over $S^{1}$ with fibre $F$ and characteristic map $h$, where $\partial F \cong K$ and $h^{m}$ is isotopic to the identity. Without loss of generality, $h$ has a fixed point $x_{0} \in \partial F$. Then $x_{0}$ gives rise to a cross-section representing a meridian $a$ in $\pi_{1}\left(X, x_{0} \tilde{\times} 0\right)$, and

$$
\pi_{1}\left(X, x_{0} \tilde{\times} 0\right) \cong \pi_{1}\left(F, x_{0}\right) \times_{h_{*}} \mathbf{Z}\langle a\rangle .
$$

Since $h_{*}^{m}=\mathrm{id}, a^{m} \in C\left(\pi_{1} X\right)$.

(7.2) THEOREM. In each dimension $n \geqslant 1$ there are knots $K_{1}^{n}, K_{2}^{n}, \ldots$ and untwisted deformations $\gamma_{i}$ of $K_{i}^{n}$ such that

(a) $\tau \gamma_{i} K_{i}^{n}$ has the same group as the $(6 i+1)$-twist-spun trefoil; and

(b) $c\left(\tau \gamma_{i} K_{i}^{n}\right)=0$.

REMARK. The knots $\tau \gamma_{i} K_{i}^{n}$ are all distinct by (a); (b) implies by Lemma 7.1 that $\tau \gamma_{i} K_{i}^{n}$ is not a twist-spun knot.

Proof. First note that it suffices to prove the case $n=1$; examples in higher dimensions can then be constructed by $p$-spinning the knots $K_{i}^{1}$ and deformations $\gamma_{i}$ as in Example 2.6. (One must check that the $p$-spin of $\tau$ is again $\tau$; we leave this to the reader.)

Now consider the 1-twist $l$-roll spin of any 1-knot $K$. Let $K$ have group $G$ and let $(\mu, \lambda)$ be a meridian-longitude pair for $K$. Let

$$
H=\pi_{1} S^{3}(K, 1 / l) \cong G /\left(\mu \lambda^{l}=1\right),
$$

and let $\varphi: G \rightarrow H$ be the quotient homomorphism. According to Corollary 5.3, $\tau \rho^{l} K$ has group $H \times \mathbf{Z}\langle t\rangle$, and a meridian is $\varphi(\mu) \times t$. Hence

$$
\pi\left(\tau \rho^{l} K\right) / C\left(\pi\left(\tau \rho^{l} K\right)\right) \cong H / C(H)
$$

and $c\left(\tau \rho^{l} K\right)$ is the order of $\varphi(\mu)$ modulo $C(H)$. 


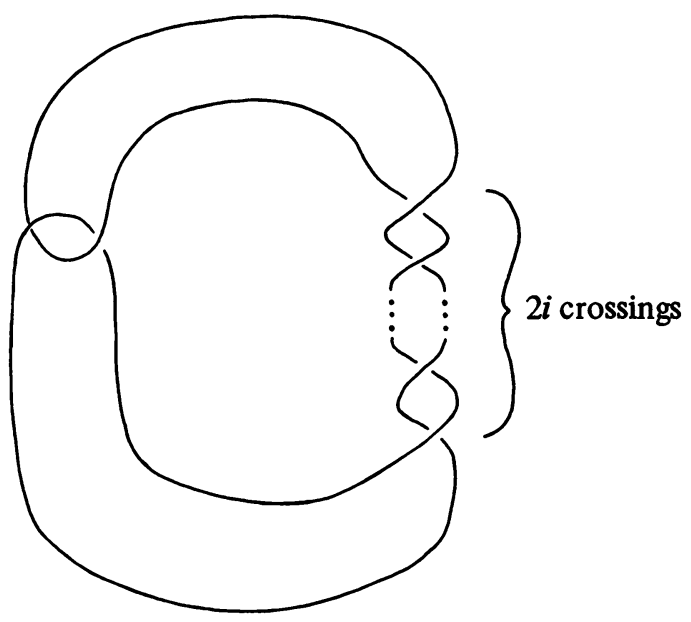

FIGURE 2

Now for $i=1,2, \ldots$ let $K_{i}$ be the knot shown in Figure 2, and let $H_{i}=\pi_{1} S^{3}\left(K_{i}, 1\right)$. The fibre $S^{3}\left(K_{i}, 1\right)$ of $\tau \rho K_{i}$ may also be obtained by surgery of type $-1 / i$ on the trefoil knot $\kappa_{2,3}$; see Figure 3 . This is the fibre of $\tau \rho^{-i} k_{2,3}$, which by Corollary 6.4 is the same as $\tau^{6 i+1} k_{2,3}$. Hence (a) holds if $\gamma_{i}=\rho$, by Corollary 5.3 .
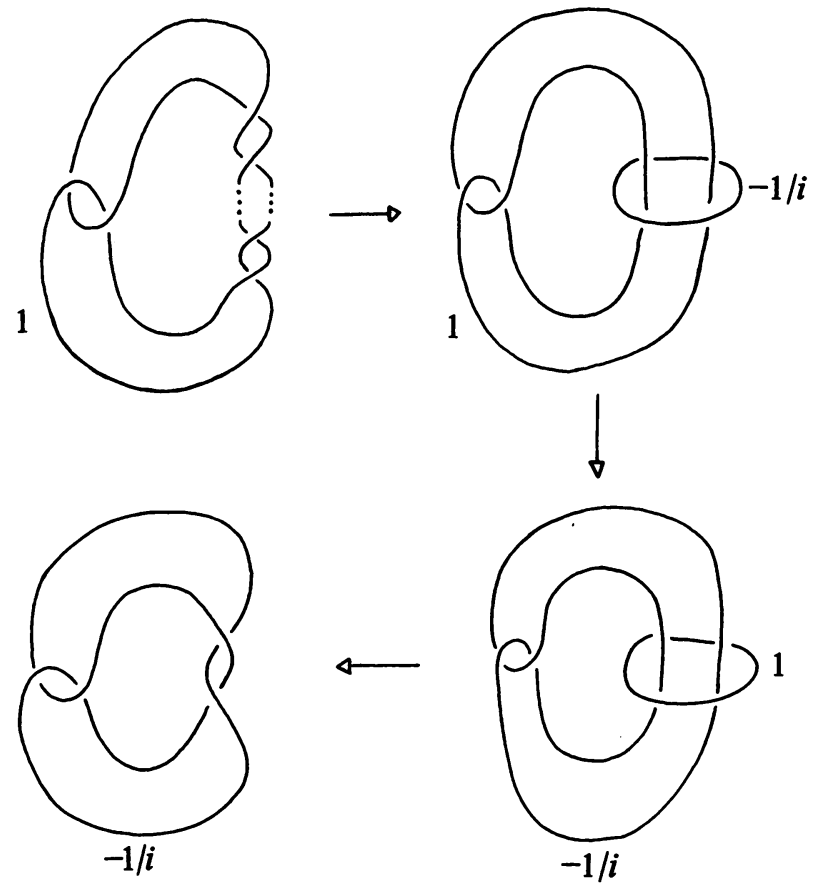

FIGURE 3 
We will use the presentation of $H_{i}$ given by the presentation $\langle a, b$ : $\left.a^{2}=b^{3}\right\rangle$ of the group of the trefoil. A meridian is $b^{-1} a$, and a longitude $a^{2}\left(b^{-1} a\right)^{-6}$. Thus

$$
H_{i}=\left|a, b: a^{2}=b^{3},\left(b^{-1} a\right)^{6 i+1} a^{-2 i}=1\right| .
$$

$C\left(H_{i}\right)$ is infinite cyclic generated by $a^{2}=b^{3}$, so

$$
\begin{aligned}
\Sigma_{i}=H_{i} / C\left(H_{i}\right) & =\left|a, b: a^{2}=b^{3}=1=\left(b^{-1} a\right)^{6 i+1} a^{-2 i}\right| \\
& =\left|a, b: a^{2}=b^{3}=\left(b^{-1} a\right)^{6 i+1}=1\right| \\
& =\left|a, b, c: a^{2}=b^{3}=c^{6 i+1}=a b c=1\right| .
\end{aligned}
$$

Groups of this form are called triangle groups, and have been extensively studied; see e.g. Milnor [9].

Now, from Figure 3 we see that a meridian of $K_{i}$ maps to the curve in $S^{3}\left(\kappa_{2,3},-1 / i\right)$ depicted in Figure 4. Recalling that the presentation $\langle a, b$ : $\left.a^{2}=b^{3}\right\rangle$ comes from the decomposition of $S^{3}$ into two solid tori with $\kappa_{2,3}$ on their common boundary (Figure 5), we see that the curve of Figure 4 represents $b a^{-1} b^{-1} a$ in $H_{i}$. Hence $c\left(\tau \rho K_{i}\right)$ is the order of the element $d=b a^{-1} b^{-1} a=b a c$ of $\Sigma_{i}$.

The proof is completed by Lemma 7.3 below.

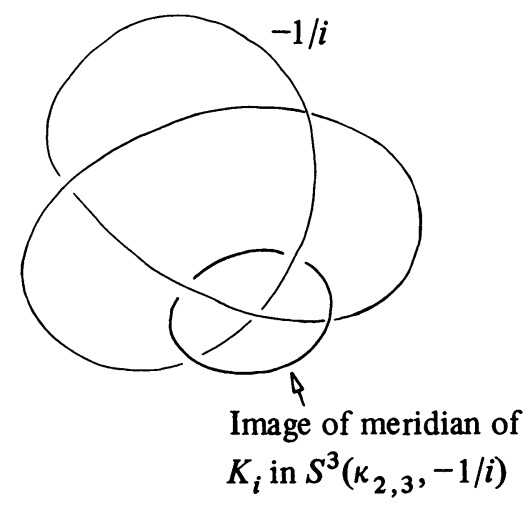

FigURE 4

(7.3) Lemma. Let $\Sigma$ be the triangle group $\left|a, b, c: a^{2}=b^{3}=c^{r}=a b c=1\right|$ where $r$ is an integer, $r>7$. Let $d=b a c$. Then $d$ has order zero in $\Sigma$.

Proof. According to Milnor [9], $\Sigma$ can be represented as the group of isometries of the hyperbolic plane $P$ generated by rotations through $\pi, 2 \pi / 3$ and $2 \pi / r$ about the vertices $A, B, C$ of a triangle with angles $\pi / 2, \pi / 3$ and $\pi / r$. Reflecting the triangle $A B C$ in its sides generates a tesselation of $P$ invariant under $\boldsymbol{\Sigma}$. A fragment of this tesselation is represented in Figure 6. 


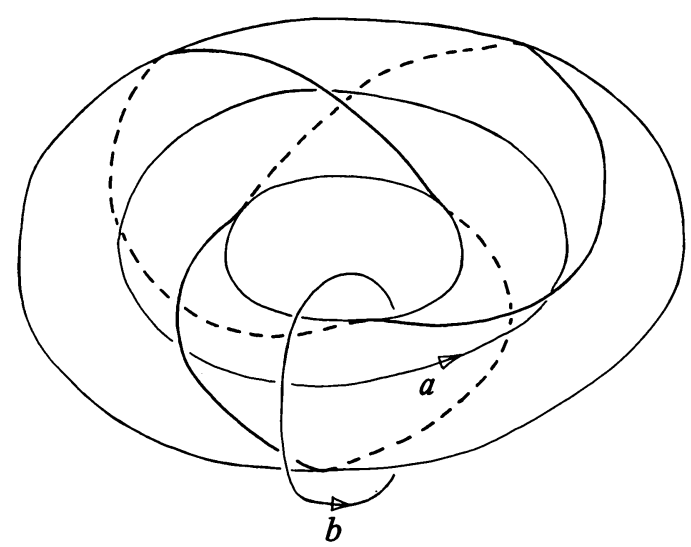

FIGURE 5

(Note: Figure 6 is actually part of the tesselation of the Euclidean plane by triangles with angles $\pi / 2, \pi / 3$ and $\pi / 6$. Hence straight lines in the diagram through points like $A$ and $B$ represent straight lines in $P$, although lines through points like $C$ do not.)

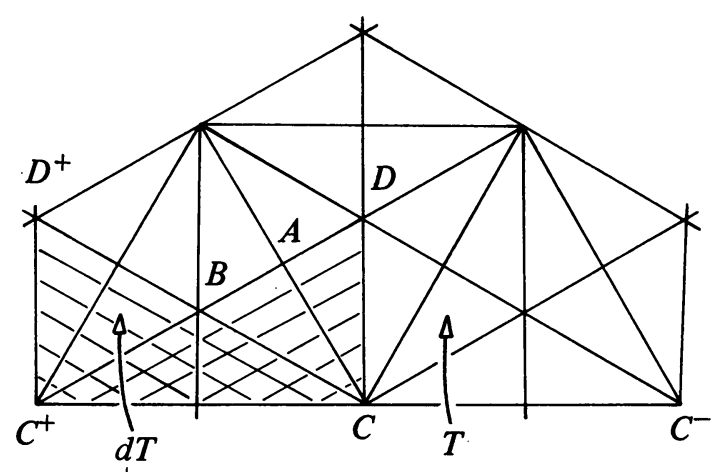

FIGURE 6

The effect of $d$ on a typical triangle $T$ is shown in Figure 6. It follows that $d$ takes the points $C^{-}, C, D$ to $C, C^{+}, D^{+}$, respectively. Suppose $d$ has nonzero order. Then it is a rotation about some point $E$, say, of $P$. Now $E$ must lie on the bisector, $C D$ produced, of $C^{-} C C^{+}$, and also on $C^{+} D^{+}$produced. Since $\widehat{C+C D}=C C^{+} D^{+}=3 \pi / r$, the triangle $C C^{+} E$ has area

$$
\pi-\left(3 \pi / r+3 \pi / r+\widehat{C E C^{+}}\right)<\pi(1-6 / r) .
$$

But $C C^{+} E$ contains the union of the triangles $C C^{+} D^{+}$and $C^{+} C D$ (shaded in Figure 6), which has area

$$
6 \pi(1-(1 / 2+1 / 3+1 / r))=\pi(1-6 / r) .
$$

This contradiction establishes the lemma. 
ACKNowledgements. I would like to thank my supervisor, Dr. C. McA. Gordon, for suggesting the problem, and for much help thereafter. I wish also to thank Professor L. Siebenmann for pointing out to me that the 1-twist 1-roll spun figure eight has the same fibre as the 1-twist $(-1)$-roll spun trefoil and asking whether these are the same knot, thus generating $\$ 7$.

\section{REFERENCES}

1. Marshall M. Cohen, A general theory of relative regular neighbourhoods, Trans. Amer. Math. Soc. 136 (1969), 189-229.

2. R. H. Fox, Rolling, Bull. Amer. Math. Soc. 72 (1966), 162-164.

3. C. H. Giffen, Homeotopy groups of fibred knots and links, Notices Amer. Math. Soc. 13 (1966), 327. Abstract \#632-10.

4. Herman Gluck, The embedding of two-spheres in the four-sphere, Trans. Amer. Math. Soc. 104 (1962), 371-383.

5. Deborah L. Goldsmith, Motions of links in the 3-sphere, Bull. Amer. Math. Soc. 80 (1974), $62-66$.

6. __ Symmetric fibered links, Ann. of Math. Studies, No. 84, Princeton Univ. Press, Princeton, N. J., 1975, pp. 3-23.

7. C. McA. Gordon, Some higher-dimensional knots with the same homotopy groups, Quart. J. Math. Oxford Ser. (2) 24 (1973), 411-422.

8. Mitsuyoshi Kato, $A$ concordance classification of PL homeomorphisms of $S^{p} \times S^{q}$, Topology 8 (1969), 371-383.

9. J. Milnor, On the 3-dimensional Brieskorn manifolds $M(p, q, r)$, Ann. of Math. Studies, No. 84, Princeton Univ. Press, Princeton, N. J., 1975, pp. 175-225.

10. Dale Rolfsen, Knots and links, Math. Lecture Series 7, Publish or Perish, Berkeley, Calif., 1976.

11. Jonathan Simon, Roots and centralizers of peripheral elements in knot groups (preprint).

12. F. Waldhausen, Irreducible 3-manifolds which are sufficiently large, Ann. of Math. (2) 87 (1968), 56-88.

13. C. T. C. Wall, Locally flat PL submanifolds with codimension two, Proc. Cambridge Philos. Soc. 63 (1967), 5-7.

14. E. C. Zeeman, Seminar on combinatorial topology, Inst. Hautes Études Sci. mimeographed notes, 1963.

15. __ Twisting spun knots, Trans. Amer. Math. Soc. 115 (1965), 471-495.

Department of Pure Mathematics and Mathematical Statistics, Cambridge University, 16 Mill Lane, Cambridge, England 